\title{
Research on Mini Desk Fan Design Based on Experimental Analysis and Numerical Simulation Technology
}

\author{
Tianzeng $\mathrm{Li}^{1, *}$, Jiajing Chen ${ }^{1}$, Hongmei Huang ${ }^{1}$, Tianyang $\mathrm{Li}^{2}$ and \\ Chunmin $\mathrm{Lai}^{1}$ \\ ${ }^{1}$ School of Industrial Design and Ceramic Art, Foshan University, Foshan 528000 \\ ${ }^{2}$ School of Marxism, Guangdong University of Foreign Studies, Guangzhou 510006 \\ *Corresponding author
}

Keywords: Industrial design, Experimental analysis, Numerical simulation technology, Mini desk fan.

\begin{abstract}
In order to realize the function of short-term rapid cooling and long-term soothing refrigeration of mini desk fan, an innovative design solution of mini desk fan based on experimental analysis and numerical simulation technology is proposed. Firstly, the market investigation and information retrieval of the design research object are carried out to determine the design goal; secondly, the performance characteristic of the existing products is analyzed with the experimental test and numerical analysis technology, and the preliminary design direction is clarified; then, the feasibility hypothesis is put forward and verified according to mechanism analysis about the previous design direction; finally, the product appearance design is driven based on the core functional characteristics of the design solution, and the final design concept is output. This design and research strategy can provide reference for similar household appliance innovation design and development.
\end{abstract}

\section{Introduction}

Improving the user experience, guiding healthy, comfortable and sustainable development attitude towards life is the main direction of modern household appliances design. Taking fan as an example, silence, low energy consumption, wireless power storage and intelligent control are the main design points[1]. Min fan is welcomed by the majority of consumers because of its lightweight and portable characteristics. The R \& D of min fan is an important business of many home appliance design companies.

At present, the research on min fan mainly focuses on the analysis of noise reduction mechanism of fan blade. For example, Cheng et al.[2] analyzed the noise characteristics of fan tip clearance and inclined /swept blade by using the mixed flow field / sound field (CFD/AA) model calculation method, and confirmed the noise reduction effect of inclined / swept static blade. Zhai et al.[3] and $\mathrm{Xiao} \mathrm{Hu}[4]$ discussed and analyzed the noise reduction performance of unequal distance blade fan. The results show that unequal distance blade fan can reduce discrete noise in the range of human ear sensitivity to noise. In the study by Qian[5], from the point of view of fan blade modeling, analyzed the effects of tail edge tooth profile, blade perforation and different tip clearance characteristics on the performance and noise of small axial flow fan. The results show that the noise reduction effect of tooth tail edge fan is better. In addition, the research object of noise reduction mechanism of min fan also involves muffler[6], support frame [7] and so on. As one of the important indexes of fan performance, the study of fan comfort is also very important. In the research of household electric fan, BALMUDA puts forward the solution of double-layer structure blade comfort fan, which represents the product of The GreenFan, which pulls the outer fast wind convergence through the inner slow wind, and then impinges each other to eliminate the vortex to achieve and the disorder effect and the purpose of simulating comfortable natural wind. However, there is a lack of research on the comfort of min fans in the field of design. In this paper, based on the existing min fan design, combined with experimental analysis and numerical simulation technology, a min desk fan product 
design solution with short-term rapid cooling and long-term soothing refrigeration function is proposed.

\section{Product Design Process Based on Experimental Analysis and Numerical Simulation Technology}

The transformation from the conceptual function of the product to the actual function is the core that determines whether the conceptual design can be successfully transformed into the real product, and it is also the most challenging link in the product design process. In order to ensure the smooth transformation of the conceptual function of the product from virtual image to reality, experimental test and analysis is an important way of presentation at this stage. The experimental analysis method has the characteristics of strong intuition and reliable results. In the experimental part of the product, the conceptual design of the product is verified one by means of physical testing. Based on the test results, the effectiveness of the structure and function of the product conceptual design can be evaluated comprehensively and concretely, the feasibility of the design can be clarified and an important reference basis for the optimization and improvement of the design can be provided. However, the experimental test method has the weakness of high cost, long cycle, and that most experimental research can only focus on the analysis and reasoning of the experimental data, but can not make persuasive explanation of the essential mechanism of the phenomenon $[8,9]$.

In recent years, as a new method of product development and analysis, computer numerical simulation technology has attracted more and more researchers' attention. The numerical simulation technology can be understood as using computer to do experiments. Compared with the traditional test methods, this method has the advantages of short research cycle and low experimental cost, and can realize the rapid evaluation and reflection of different calculation conditions. In addition, the method also has the characteristics of comprehensive calculation parameters and visual analysis of the calculation results, which provides great convenience for explaining the working mechanism of the product. At present, computer simulation technology has been widely used in aerospace, transportation, marine engineering and other fields, which provides a strong support for explaining major scientific problems and solving the bottleneck problems left over in the project[10]. In product design, numerical simulation technology can provide technical support for the development of all kinds of products, from train low resistance modeling design[11], to toaster energy saving design[12], and is gradually becoming the core technology of industrial product exploration, design, testing and operation.

The combination of experiment and numerical simulation can form complementary advantages, and it is an important means to quickly verify and accurately explain engineering and scientific problems[13]. The comprehensive application of experiment and numerical simulation technology in product design can make the design flow based on orderly and reasonable planning layout and scientific demonstration, ensure the rationality of the design process, and achieve the purpose of improving the design efficiency and quality. In this paper, the working mechanism of min fan is discussed by means of experiment and numerical analysis, which guides the reasonable design direction and driving modeling design of the product, and finally outputs the reasonable design scheme. The specific steps of product design process based on experimental analysis and numerical simulation technology are as follows:

- Concept preparation stage: problem presentation. According to the feedback of market and related information, the design goal in the early stage of the scheme can be put forward through the demand analysis of each element in the integrated product system.

- Concept brewing stage: exploration and analysis. The experimental analysis and numerical simulation model are built, the working characteristics of the existing products are analyzed, and the preliminary design direction is put forward.

- Concept formation stage: solving the problem. According to the preliminary design direction, the working mechanism is analyzed deeply, the solution is put forward, the feasibility is demonstrated and the reasonable scheme is outputted. 
- Concept output stage: design expression. Follow the principle of formal service function, choose the concept through requirement analysis, and finally output the design concept. As shown in Figure 1.

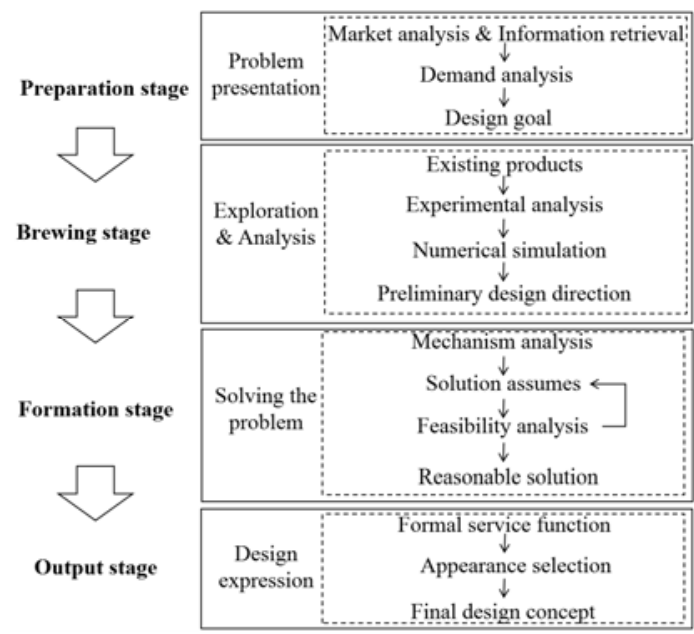

Figure 1. Product design process based on experimental analysis and numerical simulation technology.

\section{Design and Research of Min Desk Fan}

\section{Design Project Preparation}

Min desk fan is a kind of household appliance which uses motor to drive fan blade to rotate and cut air flow to accelerate air circulation. Because of its small size, convenient charging, easy to carry, moderate price and other characteristics, it has become a necessary equipment for many consumers to travel and at home for cooling and relieving heat.

On the market, the quiet characteristics of small fans are the main selling points of major fan brands. At present, the research on noise reduction of small fan mainly focuses on the characteristics of fan blade, the analysis of motor shock absorption effect, and the design of fan guard these three aspects. From the aspect of fan blade, the number of fan blade, material, shape and angle of small fan have important influence on quiet effect. From the aspect of motor shock absorption effect, material, $\mathrm{DC} / \mathrm{AC}$, frequency conversion/fixed frequency play a major role in noise reduction. From the aspect of fan guard design, the noise reduction effect can be realized in accordance with the modeling of streamlined grilles. In addition to quiet performance, the comfort of blowing is also an important index of small fan performance. The evaluation index of fan comfort includes air volume, wind pressure, wind type and so on, which is also affected by fan blade modeling, motor speed control and fan guard modeling. It is worth noting that the comfort of small fans is relative and to a large extent related to the user comfort experience. For example, for users who need to cool down quickly, the comfort can be reflected in the rapid heat dissipation and large air volume, high wind pressure indicator; For users who blow cool for a long time, comfort is reflected in low wind pressure, simulated natural wind and other indicators.

Fan blade and motor are not only the core components of desk fan, but also widely used in major household appliances, construction machinery, aerospace fields. For their key performance such as noise reduction, energy saving and so on, the related research is very rich $[14,15]$. The optimal design of the blades and motors of small fans has become mature at present, the cost of R \& D is high and the room for improvement is small. Based on the above analysis, the optimal design value of fan blade and motor to desk small fan tends to be not high. Based on the cost positioning of small fan and the current situation of lack of research on fan guard, it is more reasonable to improve the performance of min desk fan by improving the design of fan guard. 


\section{Design Project Brewing}

Classification of Existing Products. The min fan guard on the market has a variety of shapes. According to the characteristics of the fan guard structure type, the min fan guard can be roughly classified into six types, as shown in Table 1.

Table 1. Types of Small Fan fan guard

\begin{tabular}{|c|c|c|c|}
\hline Serialnumber & Representative products & Fan guard model & Structure characteristic \\
\hline No. 1 & & & Uniform linear structure \\
\hline No. 2 & & & Linear grid structure \\
\hline No. 3 & & & $\begin{array}{c}\text { Concentric circular radiation } \\
\text { structure }\end{array}$ \\
\hline No. 4 & & & Linear radiation structure \\
\hline No. 5 & & & Lurved cyclone structure \\
\hline No. 6 & & & \\
\hline
\end{tabular}

Experimental Testing. In order to intuitively understand the influence of all kinds of fan guards on the working performance of fans, this study is devoted to the transformation of No. 6 machine and the construction of a general test platform for the performance of min fans. The fan guard of each type is of uniform size, and 3D printing technology is adopted to make the test model (Table 1 fan guard model). In this experiment, the noise and wind speed of fans with different types of fan guards were recorded by noise meter and anemometer. The monitoring points were set at $0.3 \mathrm{~m}$ from the front of the fan (monitoring point 1) and 30 degrees from the middle of the fan with the same projection distance (monitoring point 2).

Based on the characteristics of external propagation of sound radiation, the size of received sound is mainly related to the length of distance. Monitoring point 1 is selected as the main data acquisition point for noise analysis. Figure 2 shows the noise performance of six fans with different fan guards at different speeds. The results show that when the fan speed is $2100 \mathrm{r} / \mathrm{min}$, the noise of each fan is about $57 \mathrm{~dB}$. When the fan speed is $2600 \mathrm{r} / \mathrm{min}$, the noise of each fan is about $65 \mathrm{~dB}$, and the influence of the change of fan guard on the noise is very limited. Figure 3 shows the variation of wind speed at monitoring point 1 and monitoring point 2 at different speeds of six fans with different fan guards. The results show that the test results of fans with different fan guards are quite different under the same test conditions.

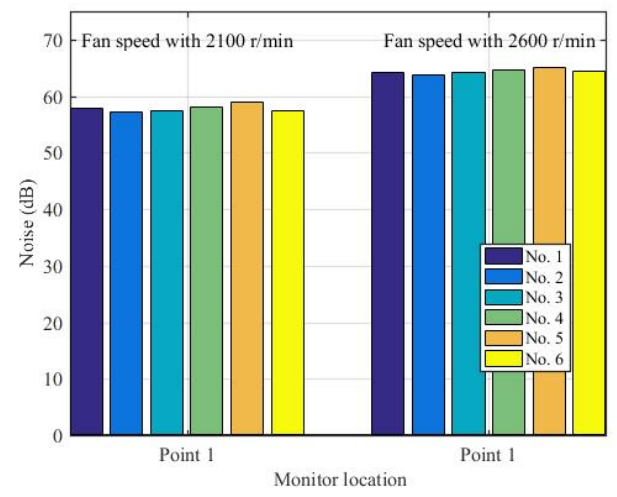

Figure 2. The noise performance of six fans with different fan guards. 


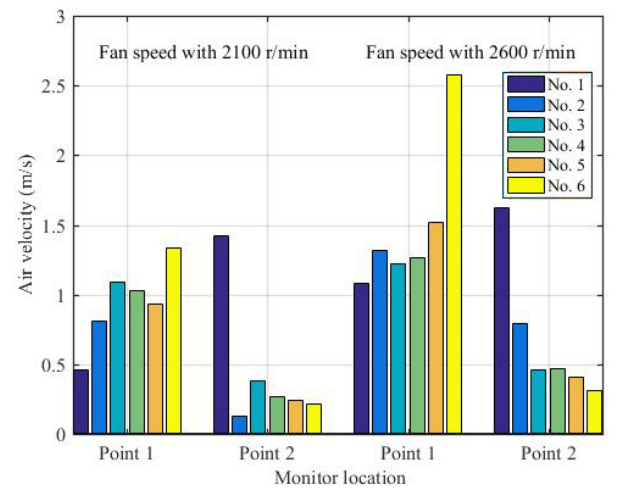

Figure 3. The variation of wind speed between monitoring point 1 and monitoring point 2 at different speeds of six fans with different FAN GUARD.

Numerical Analysis. In order to further discuss the wind field characteristics of the existing fan guard style, this study establishes the three-dimensional numerical model of the fan by means of CFD technology to realize the virtual simulation and visual analysis of the flow field.

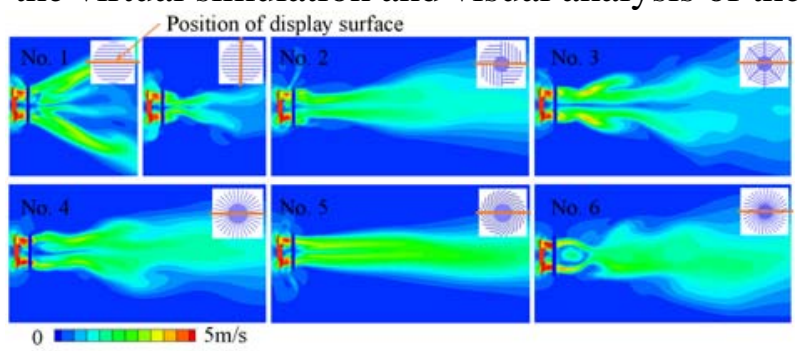

Figure 4. The wind field of fans with different types of fan guard

Figure 4 shows the wind field of fans with different types of fan guard when the fan speed is $2100 \mathrm{r} / \mathrm{min}$. It can be observed from the figure that the six fans with different types of fan guard have different wind field distribution characteristics. Among them, the fan wind field with No. 1 to No. 4 and No. 6 fan guards shows different degrees of divergence. It is worth noting that unlike the fans with other fan guards, the fans carrying No. 5 model have strong wind force and relatively weak divergence effect.

Preliminary Design Direction. According to the above analysis, the influence of the change of the fan guard on the noise reduction performance of the fan is slight, and it is not feasible to improve the quiet effect of the fan by optimizing the shape of the fan guard. However, it is worth noting that the change of fan guard has a great influence on the intensity and type of fan blowing. In particular, the fan with No. 5 fan guard shows the characteristics of high wind speed and strong convergence force, which can be used to realize the demand of rapid cooling. In addition, the fans with other fan guards have good characteristics of low wind speed and divergence, which are in line with the characteristics of natural wind. Therefore, the design point of this study can focus on how to control the switching between strong wind and natural wind to improve the applicable range and comfort of the min desk fan by improving the structure and function of the fan guard.

\section{Design Project Formation}

Mechanism Analysis. Again, the modeling characteristics of six fan fan guards can be classified, and it can be found that the grilles of No. 4, No. 5 and No. 6 fan guards are all radioactive. The clockwise adjustment of the fence of No. 6 fan guard can be changed to No. 4 fan guard, and further adjustment will become a clockwise rotating fan guard similar to the No. 5 fan guard. In order to explore the influence on the characteristics of wind field in the process of this change, by adjusting the arrangement direction of No. 6 grilles, a new clockwise rotating fan guard similar to No. 5 fan guard is established and tested. The results of Figure 5 show that the grilles of the fan guard changes from counterclockwise arrangement to clockwise arrangement, and the flow field of the original divergence disorder changes to a strong convergence wind, which is similar to that of the No. 6 fan 
guard. It can be concluded that the arrangement of the fan guard in the same rotation direction (clockwise) as the fan blade has the effect of enhancing the convergent wind field, on the contrary, it can counteract the vortex divergence wind field.

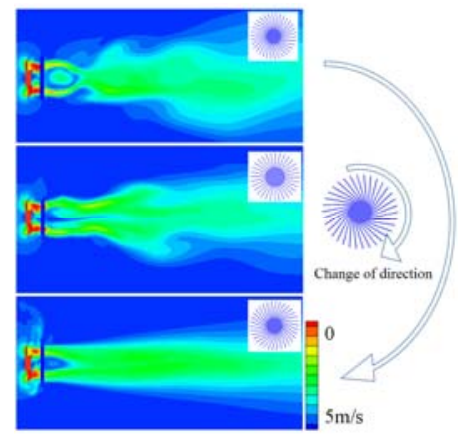

Figure 5. The influence on the characteristics of wind field by adjusting the arrangement direction of the grilles.

Propose Solutions. From the brewing stage of the design project, it can be concluded that the main problem of min desk fan design in this study is how to realize the function of blowing both strong wind and natural wind by changing the structure of fan guard. According to the analysis of the mechanism of the fan guard for the fan blowing performance, it is shown that the clockwise arrangement of the grilles can achieve the effect of fan blowing strong wind, on the contrary, it can counteract the vortex of wind dissipation and simulate the effect of natural wind. Therefore, the deformable fan guard, which can freely adjust the rotating arrangement direction of the grilles according to the specific use of the scene, is an effective way to solve the switching function between the strong wind and the natural wind of the min desk fan.

Demonstration and Final Solution. In order to realize the goal of rotating deformation of the grilles, the spoke, which is the core part of the grilles, can be selected as the soft adhesive spoke with elastic deformation, or the rigid telescopic rod spoke and the rigid hydraulic rod spoke, which can be overlapped with two pipe bodies. However, the soft adhesive spoke driven by air flow may cause vibration to increase the additional noise. Using rigid hydraulic rod as spoke miniaturization technology is complex and the cost is high. These two spoke strips are not in accordance with the performance and cost expectations of min fans. The rigid telescopic rod mechanism is simple, the miniaturization technology is mature, the structure is stable, the cost is low, it is widely used in daily products such as umbrella, clothes drying rack, selfie rod and so on, which is suitable for the design goal of small fan. Therefore, the rigid telescopic spokes are used as the final solution to realize the deformation function of the fan guard. In this solution, the grilles of the fan guard is set up with two pipe bodies overlapping, and the rigid spoke is connected with the inner ring with a hinge. The telescopic movement of the spoke is driven by the rotation of the inner ring relative to the outer ring, and the clockwise rotation effect of the fan guard is achieved, as shown in Figure 6.

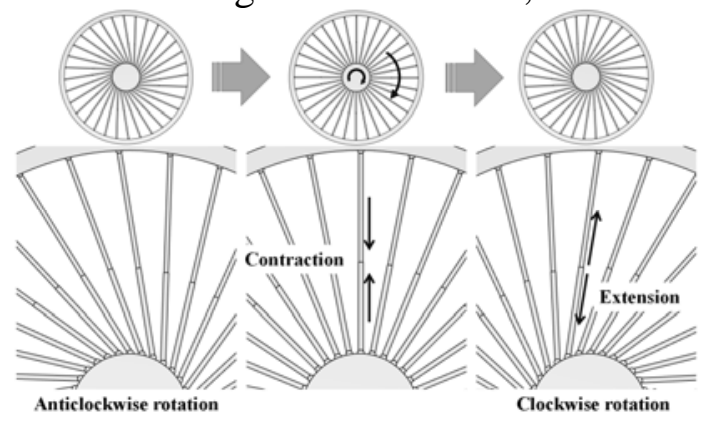

Figure 6. Deformation process of FAN GUARD.

\section{Design Project Output}

Discussion on Form and Function. In this design, the principle of telescopic movement of rigid telescopic bar spokes is used to adjust the wind guide angle of the fan guard (the grilles is switched in a clockwise or counterclockwise direction) to achieve the effect of ventilation and divergence of fan blowing. The deformable characteristics of fan mesh cover are the core factors that affect the 
appearance design of min desk fan. Around this factor, the appearance design of the min desk fan needs to solve the following problems:

- The relationship between the structural characteristics of the rotatable deformation of the fan guard and the overall appearance of the fan;

- The human-computer interaction design problem which can realize the control button of the rotating deformation of the fan guard.

Selection of Appearance and Color. According to the overall modeling design of fan, on the one hand, the market of household appliances is mainly sales of circular fan, and the circular modeling design is in line with the characteristics of fan blade rotation periodicity, and its rotation path is symmetrical, which can unify the average length of rigid spoke and the intensity of telescopic change, which is beneficial to the switching of clockwise and counterclockwise rotation angle of fan guard. On the other hand, the circular modeling design curve is soft, easy to shape a simple design style, transfer natural and simple design concept. Therefore, in this study, the circle was selected as the overall shape of the min fan.

Aiming at the design level of fan control knob appearance, based on the integrated design concept of control knob and circular fan guard, the knob design solution (shown in Figure 7) is put forward, and from the point of view of convenience of interaction mode, the design scheme of circular rotation control knob in the center of fan guard is adopted.

The color and material of the product is related to the orientation of the consumer population. This design takes the positioning of the middle and high-end consumers as an example. The shell and bracket are mainly made of the cold-grey abrasive metal material that reflects the modern style, and the fan blade is the same color as the fan body, so as to create a quiet, stable atmosphere, and reflects the light luxury, elegant and atmospheric design style.
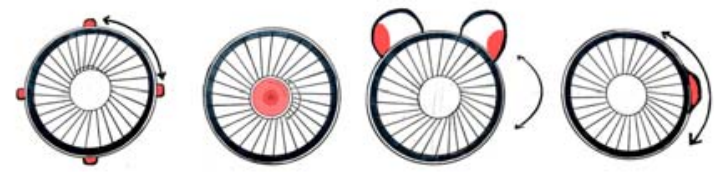

Figure 7. Some of the fan control knob design solution.

Final Conceptual Products. Through improving the details of the min desk fan solution, and the final product design effect is output, as shown in Figure 8.

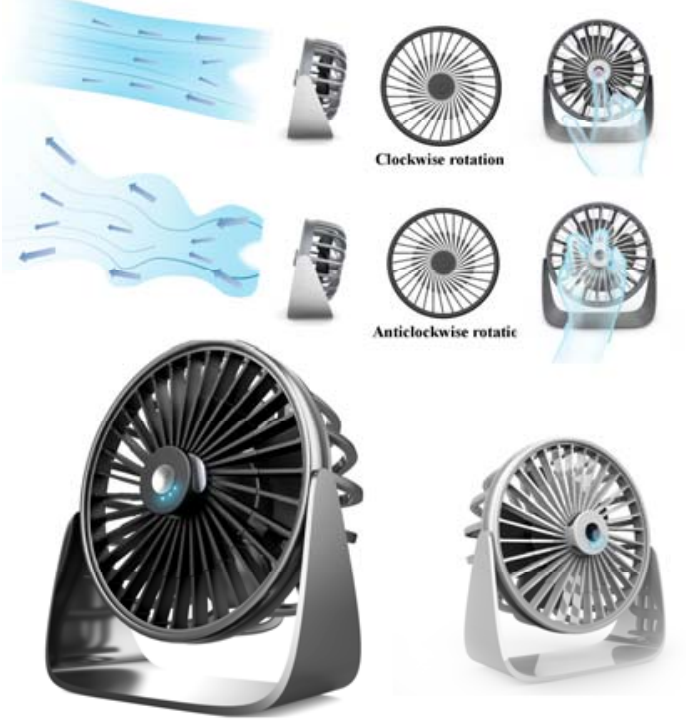

Figure 8. Deformation process of FAN GUARD. 


\section{Conclusion}

In this study, a product design process based on experimental test and numerical simulation technology is proposed. Firstly, through the preliminary investigation and analysis of the design object, the appropriate design focus point is determined. Secondly, the functional characteristics of the existing products are analyzed and summarized by means of experimental and numerical analysis, and the breakthrough point of the design is found. Then, the product mechanism is analyzed deeply, and on this basis, a reasonable solution is put forward. Finally, by discussing the relationship between form and function, the final product appearance is determined, and a complete product design concept is output. This method highlights the important role of technical analysis in the design of logical framework, can effectively overcome the blindness and contingency problems caused by product design project, and help to improve the efficiency and quality of product design. The design method proposed in this paper effectively guides the formation and output of innovative design solution for min desk fan, and can provide reference for similar product design and development.

\section{Acknowledgment}

The authors gratefully acknowledge the financial support of National Natural Science Foundation of China (NSFC) (No: 11802061) and Scientific Research Fund of Guangdong Education Department (No:2017KQNCX208).

\section{References}

[1] Z. Jiaming, "Intelligent Electric Fan Based on the Bluetooth Control of Mobile Phone," Telecom. World, Vol. 2017, pp. 282-283, March 2017. (in Chinese)

[2] C. Haoyi, Q. Weiyang, W. Liangfeng, T. Fan, and W. Xunnian, “Control of Tonal Noise in an Axial Flow Fan with Rotor Tip Clearance, Stator Lean and Sweep,” J. Propul. Technol, Vol. 40, pp. 295-306, December 2018. (in Chinese)

[3] Z. Shufeng and S G. Wenbin, "Study on Noise Reduction of Fans with Uneven Blade Spacing," Sci. Technol. Vis, Vol. 2018, pp. 138-140+146, August 2018. (in Chinese)

[4] H. XiaoWanyue, "Study on Aerodynamic Performance Calculation and Noise Reduction of Fans with Uneven Blade Spacing,” S. China. U. Technol, June 2018. (in Chinese)

[5] Q. Hongyu, "Research on Influencing Factors about Small Axial Flow Fan's Performance and Noise,” Zhejiang. Sci-Tech. U, March 2012. (in Chinese)

[6] Y. Mingsui, L. Dong, and Z. Lei, "Analysis on the design and noise reduction of a certain type of a fan exhaust muffler,” J. Shenyang. Aerosp. U, Vol. 33, pp. 15-22, December 2016. (in Chinese)

[7] W. Kexin, J. Yingzi, L. Yi, and C. Wei, "Study on the Structure Parameters Optimization of Struts of Small Axial Flow Fan,” J. Zhejiang. Sci-Tech. U(Nat. Sci. Edit), Vol. 37, pp. 381-388, January 2017. (in Chinese)

[8] L. Tianzeng, C. Wenhao, and Z. Jiemin, “Numerical Investigation of Swimmer's Gliding Stage with 6-DOF Movement,” PloS. One, Vol. 12, pp. e0170894, January 2017.

[9] L. Tianzeng and Z. Jiemin, "Hydrodynamic body shape analysis and their impact on swimming performance,” Acta. Bioeng. Biomech, Vol. 17, pp. 3-11, June 2015.

[10] S. C. Chapra and R. P. Canale, “Numerical methods for engineers,” New York: Mcgraw-hill, 1998.

[11] D. Guofu, J. Jie, Z. Haizhu, M. Xiaojie, L. Rong, Z. Yisheng, et al., 
"Development and challenge of digital design of high-speed trains in China,” J. Southwest. Jiaotong. U, Vol. 51, pp. 253-263, April 2016.

[12] L. Tianzeng, F. Minda, C. Wenhao, and H. Ling, "Application of Numerical Simulation Technology in Industrial Design:Taking the Energy-efficient Toaster Design as an Example,” Art. Des,Vol. 2018, pp. 86-89, June 2018. (in Chinese)

[13] P. Parnaudeau, J. Carlier, D. Heitz,and E.Lamballais, "Experimental and numerical studies of the flow over a circular cylinder at Reynolds number 3900,” Phy. Fl, Vol. 20, pp. 085101. August 2008.

[14] E. Envia, “Fan noise reduction: an overview,” Int. J. Aeroacoust, Vol. 1, pp. 43-64. January 2002.

[15] J. Li, X. Song, and Y. Cho, "Cho, Comparison of 12/8 and 6/4 switched reluctance motor: Noise and vibration aspects,” IEEE. Trans. Magn, Vol. 44, pp. 4131-4134. November 2008. 